\title{
Integrating Region and Edge Information for the Automatic Segmentation of Interventional Magnetic Resonance Images of the Shoulder Complex
}

\author{
Marie-Eve Tremblay ${ }^{1}$, Alexandra Branzan Albu ${ }^{1}$, Luc Hébert ${ }^{2}$, Denis Laurendeau ${ }^{1}$, \\ ${ }^{1}$ Computer Vision and Systems Laboratory, Dept. of Electrical Engineering, Laval University, Québec, G1K 7P4 \\ ${ }^{2}$ National Defence of Canada, Health Services Delivery, CS Valcartier succ FORCES,Courcelette (QC) GOA 4Z0 \\ marie-eve014@,sympatico.ca, branzan@gel.ulaval.ca, 1jhebert@,videotron.ca, laurend@gel.ulaval.ca
}

\begin{abstract}
This paper proposes a new $2 D$ segmentation method for MR shoulder images. Due to the significant length of the image sequences, we aim at minimizing the user intervention in the segmentation process. Our method integrates region and edge information in a coherent manner. In fact, the edge information is used in the definition of an adaptive similarity measure for iterative pixel aggregation. The seeds for the region growing process are defined automatically, which is essential for processing long image sequences with variable average brightness. Moreover, the proposed segmentation approach implements parallel region growing processes, and allows for dynamic region merging at successive iterations. To assess the performance of the proposed approach, we followed a standard methodology used for validating $2 D$ segmentation, as well as a quantitative and qualitative evaluation of the $3 D$ shoulder model reconstructed from the segmented image sequences.
\end{abstract}

\section{Introduction}

The visualization of musculoskeletal structures is a powerful tool for diagnosis, surgical planning, and post-operative evaluation. The recent developments in the technology of magnetic resonance image acquisition systems have opened interesting opportunities for the study of shoulder pathologies as well as for the assessment of the rehabilitation process. Specifically, the open-field architectures of MR systems with horizontal and vertical access (see Figure 1) allow for the study of the shoulder complex in different key positions during arm elevation and abduction.

Prior to the $3 \mathrm{D}$ reconstruction of the anatomical structures from 2D image sequences, a segmentation process must take place in order to identify the region of interest (e.g. bony structures, tendons, ligaments, calcifications etc.). In the context of the musculo-skeletal imagery, the segmentation of the bony structures constituting the shoulder complex must be highly accurate, since the intra-structural distance is an important factor for the diagnosis of pathologies such as the rotator cuff disease and the shoulder impingement [1].

The main goal of our work is to implement an automated segmentation algorithm of 2D MR images of the shoulder. The result of the slice segmentation will be further used to reconstruct a 3D model of the shoulder and to analyze the evolution of interstructural distances during arm elevation and abduction.
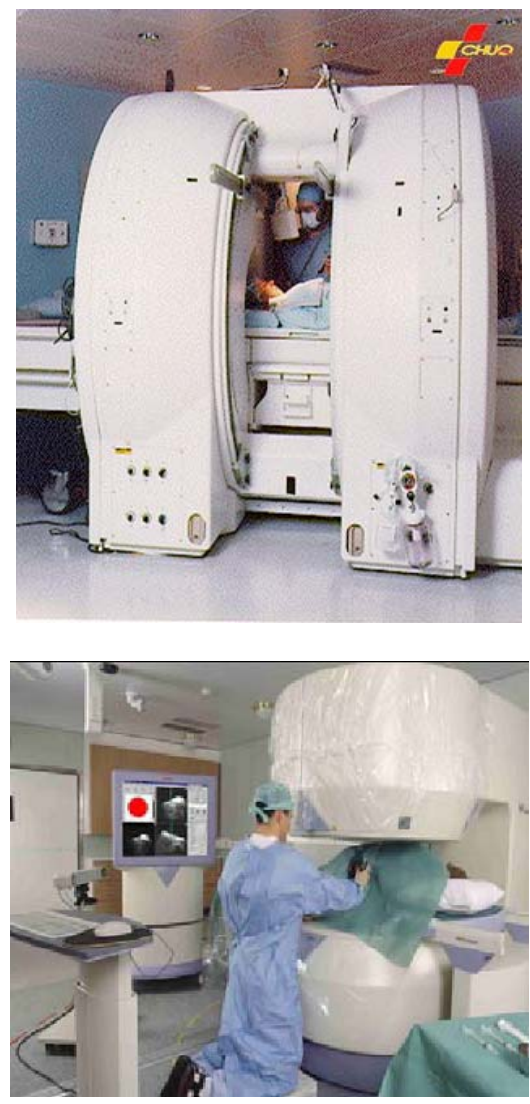

Figure 1. Open-field architectures of interventional magnetic resonance systems allowing

Since our approach is task-oriented, we were strongly motivated to investigate the specific features 
of the functional magnetic resonance images of the shoulder complex. A list of the main relevant features for our research is following.

a) The bony structures are not textured, and their appearance is dark and homogeneous in T1-weighted MR images.

b) The structure-background transition is rather smooth, which results in blurred boundaries of the regions of interest in the image. This phenomenon, called the partial volume effect [2], is caused by the finite thickness of the slices and is always present in volumetric medical imaging.

c) The average brightness exhibits a significant inter-slice variance during the same MR sequence. Due to this variability and to the large number of images contained in one MR sequence, standard segmentation approaches based on user-specified thresholds are not recommendable.

d) The complex morphology of the bony structures involved in the musculo-skeletal shoulder complex prevents from performing 3D segmentation, since the number of compact regions may vary from one slice to the next.

Considering the previously mentioned features, this paper proposes a new 2D segmentation method for MR shoulder images involving minimal user interaction. Our method integrates region and edge information in a coherent manner. The proposed segmentation technique performs a parallel region growing process using an adaptive edge-based similarity measure.

The rest of the paper is organized as follows. Section 2 briefly reviews the literature on segmentation of medical images highlighting recent research on the development of hybrid methods. Section 3 describes the proposed approach. Section 4 presents the results as well as their validation. Finally, Section 5 draws the conclusions and presents future work directions.

\section{Background}

Since the accurate detection of the region of interest is a central problem in medical applications, a large variety of segmentation algorithms are dedicated to specific types of medical images. We have identified four main types of segmentation techniques suitable for volumetric medical images.
Threshold-based approaches are usually semiautomatic and classify pixels with respect to brightness information [3][4]. These techniques present a high inter-observer variability if the threshold is specified by the user. In the case of automatic thresholding, the image histogram must contain separable modes, modeled as Gaussian distributions. There is a pairwise correspondence between the modes of the histogram and the classes detected in the image.

Boundary-based methods use gradient filters to detect inter-class separation boundaries [5]. In volumetric medical images, partial volume effect results in structures with blurred edges. Therefore, the edge detection process for the structure of interest may be incomplete. Postprocessing using contour closing approaches is possible [6], but not really practical due to the high-order computational complexity of these techniques.

Region-based methods perform pixel aggregation around user-specified seeds using homogeneity and adjacency criteria [7]. Since region growing algorithms are iterative, an additional convergence criterion must be specified [8]. Depending on the task at hand, this criterion may be expressed as a maximal acceptable difference between the brightness of the candidate pixel and the average brightness of the grown region, or may be related to the size and shape of the region. Region growing techniques are often used in medical imaging for the segmentation of homogeneous structures. In addition, user-based seed specification allows information to be extracted about the localisation of the region of interest in complex images where multiple anatomic structures are visible.

Recently, task-oriented hybrid methods have been developed, aiming at an optimal combination of the advantages offered by the three previously mentioned basic approaches. The hybrid segmentation method described in [9] combines local adaptive thresholding with region growing for the detection of skeletal structures in CT image sequences. This method requires user interaction as well as continuous input image sequences, with no missing slice and a minimal slice thickness. The hybrid method presented in [10] combines adaptive region growing with a homogeneity model. Their algorithm is rather slow, consisting in two steps: first, the homogeneity criterion is learned from the global image appearance, and second, the region of interest is grown around a user-specified seed pixel. 
A segmentation technique for 2-D interventional MR images of liver tumours is presented in [11]. This technique integrates edge and region information in order to detect textured liver tumours with blurred boundaries of non-uniform sharpness.

The following section proposes a new hybrid segmentation method combining edge detection and region growing. The edge information is used for the specification of a convergence criterion in the iterative pixel aggregation process.

\section{Proposed approach}

Our work is focused on the development of a segmentation approach for interventional MR images of the bony structures in the shoulder complex. The database contains three types of parallel planar image sequences corresponding to three different orientations of the reference plane : saggital, axial and coronal respectively. The morphology and global appearance of the bony structures varies significantly in different planes, as shown in Figure 2.

Due to the significant length of the image sequences, we aim at minimizing the user intervention in the segmentation process. Moreover, heavily user-dependent segmentation algorithms yield unstable performances, due to the large variability of the users' ability to perform a correct identification of the structures of interest in the image to be segmented. The pre-processing phase of our approach gives more details about the amount of information requested from the user.

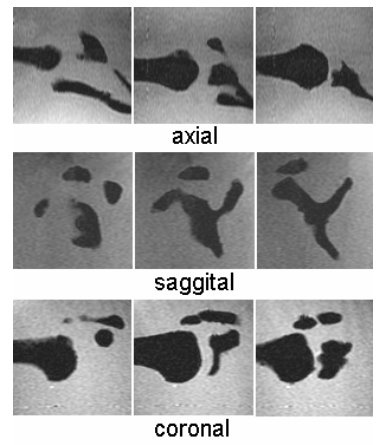

Figure 2. Relevant samples of MR shoulder images corresponding to three standard orthogonal views

\subsection{Selection of the region of interest}

A user-friendly interface is designed for the selection of a rectangular region of interest (ROI) in the image. This interface allows the user to scan the entire sequence at the desired speed, to pause for a more thorough image examination and finally to draw a rectangular region framing the structures of interest throughout the entire sequence. The ROI is drawn only on the first image of the currently analyzed sequence and automatically mapped afterwards on every subsequent image belonging to the same sequence.

The region of interest only partially encloses the bony structures involved in the shoulder complex, since the medical diagnosis is based on the analysis of the central zone. As shown in Figure 3, the inferior part of the scapula is left outside the ROI. Moreover, the dimensions of the ROI may vary from one view to another, but remain invariant for all images belonging to the same sequence.

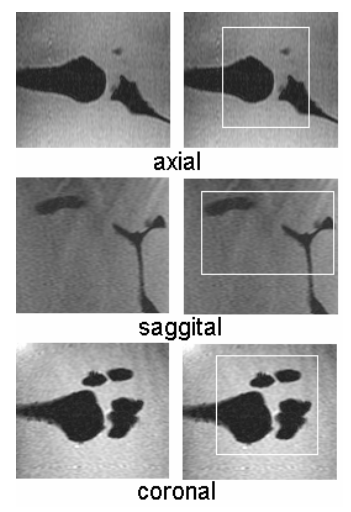

Figure 3. The ROI, enclosed in the white rectangle superposed on each image in the same sequence

\subsection{Noise reduction}

The image acquisition process introduces an undesirable component that is uncorrelated with the signal carrying the relevant information. This component is referred to as random noise. Random noise is an important limiting factor in MR imaging. It is introduced early on the signal generation and detection stage and is then processed in the image reconstruction stage.

It is well known that Wiener filtering performs well for image restoration in a noisy environment [8]. This approach integrates the degradation function associated with the signal acquisition and the statistical features of the noise occurring in the reconstruction process, in order to increase the signal-to-noise ratio (SNR). The Wiener method is founded on modelling both the uncorrupted image and the noise as random processes, and aims at finding an optimal estimate $\hat{f}$ of the image $f$. This 
optimal estimate is computed by minimizing the mean square error between $\hat{f}$ and $f$.

First, the local mean and variance around each pixel have to be estimated. For the estimation process we consider a sliding square window of size $5 \times 5$ in order to be sequentially centered at each pixel location in the image. The size of the window was selected according to a qualitative estimation of the global homogeneity of the sequences in the database. The estimated local mean and variance are computed as follows :

$$
\begin{aligned}
& \mu\left(i_{0}, j_{0}\right)=\frac{1}{25} \sum_{(i, j) \in V_{0}} I(i, j) \\
& \sigma^{2}\left(i_{0}, j_{0}\right)=\frac{1}{25} \sum_{(i, j) \in V_{0}} I^{2}(i, j)-\mu^{2}(i, j)
\end{aligned}
$$

where $\left(i_{0}, j_{0}\right)$ is the current location in the image, $\mathrm{V}_{0}$ is the $5 \times 5$ neighbourhood centered on $\left(i_{0}, j_{0}\right)$ and $I$ is the image intensity function.

Since we do not have any knowledge about the statistical noise distribution, we compute the noise variance $\sigma_{n}^{2}$ as the average of all the local estimated variances.

$$
\sigma_{n}^{2}=\frac{\sum_{i=1}^{N} \sum_{j=1}^{M} \sigma^{2}(i, j)}{N x M}
$$

where $\mathrm{N}$ and $\mathrm{M}$ are the dimensions of the rectangular region of interest.

Thorough testing on the image database has proven the efficiency of a hybrid, threshold-based filtering approach. This approach works on a pixel-by-pixel basis and considers two alternatives : a) if the local variance $\sigma^{2}(i, j)$ is lower than the noise variance $\sigma_{n}^{2}$, then the filtered intensity value is set to the local mean $\mu(i, j)$; and b) if the local variance exceeds the noise variance, then the Wiener filtering approach is implemented.

Thus, the intensity function of the filtered image has the following expression:

$$
I^{\prime}(i, j)=\left\{\begin{array}{c}
\mu(i, j) \text { if } \sigma^{2}(i, j) \leq \sigma_{n}^{2} \\
\mu(i, j)+\frac{\sigma^{2}(i, j)-\sigma_{n}^{2}}{\sigma^{2}(i, j)}(I(i, j)-\mu(i, j)) \text { otherwise }
\end{array}\right.
$$

where

- $i=\overline{1, M}, j=\overline{1, N}$
- $I(i, j)$ and $I^{\prime}(i, j)$ are the intensity functions of the original and filtered images respectively.
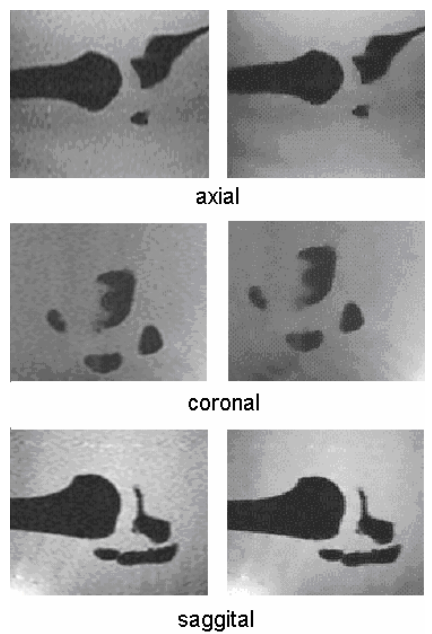

Figure 4. Left-original images; right-filtered images

Figure 4 shows the result of the filtering phase for axial, coronal, and saggital image samples respectively. The visual examination of these images proves that the global appearance of the structures of interest does not change much. However, the intrastructural texture is more homogeneous and the noise in the background is reduced as well.

\subsection{Edge detection}

The edge detection aims at an accurate extraction of the contours corresponding to the bony structures in the image. In a first step, Sobel operators [11] are used to compute the horizontal and vertical firstorder image derivatives.

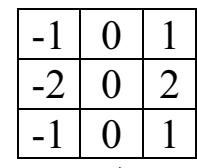

a)

\begin{tabular}{|c|c|c|}
\hline-1 & -2 & -1 \\
\hline 0 & 0 & 0 \\
\hline 1 & 2 & 1 \\
\hline
\end{tabular}

b)

\begin{tabular}{|l|l|l|}
\hline $\mathrm{I}_{1}$ & $\mathrm{I}_{2}$ & $\mathrm{I}_{3}$ \\
\hline $\mathrm{I}_{4}$ & $\mathrm{I}_{5}$ & $\mathrm{I}_{6}$ \\
\hline $\mathrm{I}_{7}$ & $\mathrm{I}_{8}$ & $\mathrm{I}_{9}$ \\
\hline
\end{tabular}

c)
Figure 5. a) Sobel mask for the horizontal derivative; b) Sobel mask for the vertical derivative; c) Labelling of the pixels to be convolved with the masks.

The convolution of the $3 \times 3$ Sobel masks (see Figure 5 ) with the original image generate one image for the horizontal derivative $(B x)$ and one image $\left(B_{y}\right)$ for the vertical derivative, as follows : 


$$
\begin{aligned}
& B_{x}\left(I_{5}\right)=\frac{1}{8}\left(-I_{1}+I_{3}-2 I_{4}+2 I_{6}-I_{7}+I_{9}\right) \\
& B_{y}\left(I_{5}\right)=\frac{1}{8}\left(-I_{1}-2 I_{2}-I_{3}+I_{7}+2 I_{8}+I_{9}\right)
\end{aligned}
$$

Since the two Sobel masks slide over the entire image, the values of the first-order partial derivatives are computed in a pixelwise manner.

The magnitude of the gradient conveys local information about the strength of the edges. The magnitude of the gradient is defined as:

$$
\nabla(i, j)=\sqrt{B x^{2}(i, j)+B y^{2}(i, j)} \quad i=\overline{1, M}, j=\overline{1, N}
$$

While low-magnitude edges correspond usually to intra-structural texture, high-magnitude edges are more likely to identify, at least partially, with the contours of the bony structures.

High-magnitude edges are selected with respect to a threshold $t_{1}$, which represents the average value of the gradient magnitude over the image:

$$
t_{1}=\frac{\sum_{i=1}^{M} \sum_{j=1}^{N} \nabla(i, j)}{M \cdot N}
$$

The extraction of high magnitude edges yields a binary image containing rather thick contours. This result is mainly due to the partial volume effect (PVE) present in magnetic resonance images and caused by the finite slice thickness during the acquisition process. This artifact results in smooth intensity transitions between structures exhibiting very different proton densities.

Our approach shrinks 'thick' edges until obtaining one-pixel width contours. Only pixels belonging to the initial 'thick' edge and representing either horizontal or vertical local maxima of the gray-level gradient magnitude are preserved in the final one pixel width contour. The decision of choosing between the horizontal and the vertical orientation is made with respect to the relative strength of the two derivatives at each pixel location. Our shrinking approach is expressed in the following equation:

$$
C(i, j)=\left\{\begin{array}{cc}
1 & \text { if }\left(B_{x}(i, j) \geq B_{y}(i, j)\right) \wedge \\
& \wedge(\nabla(i, j) \geq \max (\nabla(i+1, j), \nabla(i-1, j))) \\
1 & \text { if }\left(B_{y}(i, j) \geq B_{x}(i, j)\right) \wedge \\
& \wedge(\nabla(i, j) \geq \max (\nabla(i, j+1), \nabla(i, j-1))) \\
& 0 \text { otherwise }
\end{array}\right.
$$

where $\mathrm{C}$ is the binary image containing one pixelwidth contours, $\mathrm{B}_{\mathrm{x}}$ and $\mathrm{B}_{\mathrm{y}}$ are the gray-level images corresponding to the horizontal and vertical firstorder derivatives, and $\nabla$ is the gray-level image representing the total gradient magnitude.

Our thinning approach preserves the connectivity of the edges contained in the initial thick structures. However, the contours in the resulting image $\mathrm{C}$ are not closed (see Figure 6), due to local disconnections generated by the partial volume effect. In order to be able to perform an accurate segmentation of the regions of interest, we integrate the proposed contour detection with a region-growing method. This method will be described in the next section.

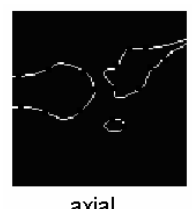

axial

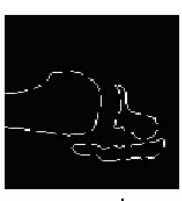

coronal

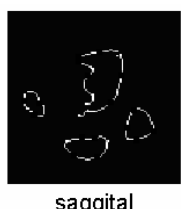

saggital
Figure 6. Contour images resulting after edge detection

\subsection{Region growing}

\subsubsection{Seed specification}

Region growing techniques are bottom-up methods and consist in pixel aggregation around an initial set of seed pixels. The pixel aggregation process iteratively adds to the region the neighboring pixels satisfying a similarity constraint, and stops when no candidate pixel satisfies this constraint.

The correct specification of the seed pixels is essential for the success of the approach. Choosing the wrong seeds may compromise the following process, since the similarity of the pixels to be aggregated is first computed with respect to the seed.

Our approach performs an automatic histogrambased selection of the seeds. The bony structures of interest in shoulder T1 MR images are homogeneous and darker than the surrounding tissues. Our approach computes the global intensity histogram of the rectangular region of interest defines the seeds as pixels with intensity values belonging to the $5 \%$ inferior range of the histogram. The mathematical expression of this condition is as follows:

$$
S(i, j)=\left\{\begin{array}{c}
1 \text { if } I(i, j) \leq I_{\min }+0.05\left(I_{\max }-I_{\min }\right) \\
0 \text { otherwise }
\end{array}\right.
$$


where $S(i, j)$ is a binary image containing the seed pixels. Figure 7 shows examples of seed images corresponding to the axial, saggital and respectively coronal sequences.

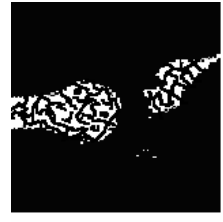

axial

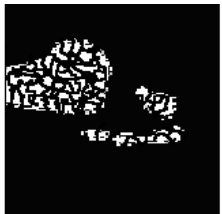

coronal

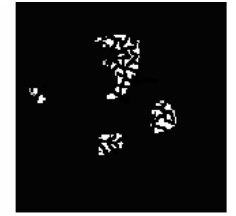

saggital
Figure 7. Binary images containing the seed pixels for the region growing algorithm

\subsubsection{Labelling of disjoint regions}

As shown in Figure 7, most of the seed pixels are distributed in small sized regions. Therefore, a labelling of compact seed regions is performed prior to the beginning of the pixel aggregation. The compactness of the seed regions is evaluated with respect to the 4-connectivity of adjacent pixels. After the initial labelling process, a set of $R_{n}$ compact and disjoint regions, $i=\overline{1, N}$ is generated.

\subsubsection{Defining the convergence criterion}

As for the seed specification, the convergence criterion is essential for the success of the region growing process. Usually, this criterion is a function related to a similarity threshold computed between the currently evaluated candidate and the corresponding instance of the growing region. The iterative pixel aggregation algorithm for a given labelled region stops when there is no candidate pixel that satisfies the similarity threshold.

We propose a new definition for the convergence criterion based on an adaptive edgebased similarity measure. For each labelled growing region $R_{n}$, a list $L_{n}$ of pixels containing relevant edge information is created as follows:

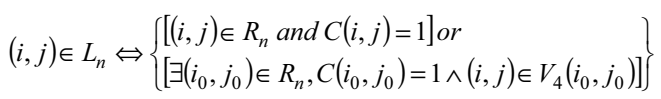

The list $L_{n}$ contains contour pixels belonging to the labelled region $R_{n}$ as well non-contour pixels adjacent to $R_{n}$ and having a contour pixel in their $\mathrm{V}_{4}$ neighbourhood. The inclusion of the non-contour pixels in the list allows us to handle the local disconnections in the contour image.

The lists are sorted in ascending order with respect to the intensity of their elements. This sorting operation allows the similarity threshold to be set to the median intensity value in the ordered list $L_{n}$. The proposed similarity measure is consistent with the specific appearance of the MR shoulder images. Thus, the peripheral zone of the bony structures is always brighter than the centre. Consequently, the defined similarity threshold is updated at each iteration of the region growing process.

\subsubsection{Insertion of the best candidate}

At a given iteration of the pixel aggregation, only one pixel is inserted in every labelled region of the image respectively. This pixel represents the best candidate among all current candidates image. To be a candidate, a pixel must satisfy the following conditions: 1) It does not already belong to a labelled region; 2) It is adjacent to an instance of a labelled region with respect to the 4-connectivity; 3) The convergence criterion is not yet satisfied.

For each labelled region, an independent list of candidates is created. The best candidate is the one having the lowest intensity value. If two candidates have the same intensity but one of them belongs to an edge in the binary contour image $\mathrm{C}$, the non-edge candidate is chosen as the best candidate. This decision is coherent with the edge-based convergence criterion, and prevents the premature ending of a labelled region growing process.

The pixel aggregation processes are parallel and independent for every labelled region. However, the iterative evolution of the processes may result in the merging of one or more labelled regions. Moreover, this merging phenomenon is predictable, since at the initial step of the process, every bony structure in the image was represented by a set of disjoint seed regions. At the convergence of the region growing algorithm, we expect to obtain a pairwise correspondence between the bony structures present in the image and the labelled regions.

If merging of two regions occurs at a given iteration of the pixel aggregation process, the two regions will receive the same label for the next iteration and will be further considered as one region. Consequently, the number of labelled regions decreases while advancing in the pixel aggregation process. After performing the inclusion of the best candidates in the corresponding labelled regions, the similarity edge-based threshold for the next iteration is computed using the updated list $L_{n}$.

The global convergence of the parallel region growing algorithm is reached when every local convergence criterion for the labelled regions is 
satisfied. As previously mentioned, the final number of labelled regions is equal to the number of bony structures present in the image. Figure 8 shows samples of segmentation results for axial, coronal and saggital slices respectively.

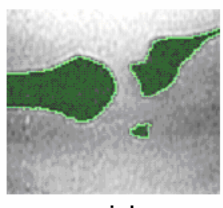

axial

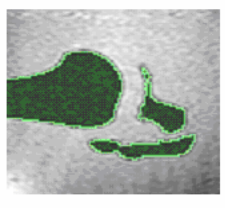

coronal saggital

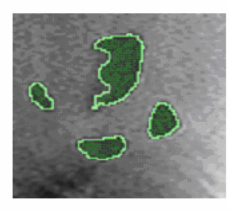

Figure 8 . Segmentation results. The regions obtained after the global convergence are highlighted in green and superposed over the original images.

\section{Results}

The database for this study contains 20 T1weighted functional magnetic resonance sequences from three different views of the shoulder: axial, sagittal and coronal. The average length of a sequence is of 30 images. The images are stored in an uncompressed format and contain 256 gray levels. The size of the images is $256 \times 256$ pixels, while the intra-slice pixel resolution is of $1.25 \mathrm{~mm}$. The value of slice thickness is set to $7 \mathrm{~mm}$, which is a reasonable trade-off between the strength of the partial volume effect and the temporal extent of the acquisition process.

The images were acquired on General Electric Sigma Horizon open-field MR system at the Intervention Magnetic Resonance Imaging Unit of the Centre Hospitalier Universitaire de Québec (CHUQ). Reference segmentations were available for every sequence in the database.

In order to evaluate the performance of the proposed segmentation approach, we followed two procedures. The first one is a standard methodology used in the validation of 2D segmentation techniques [12]. The results obtained by our approach were compared to the manual segmentation performed by a radiologist. Conventional editing tools in a webbased graphics custom-made interface allowed for a rapid information exchange with the radiology group.

Let us consider $S$, a compact object generated with our segmentation approach and $R$, the reference object enclosed by the manually edited contour. The surface overlap metric in [12] performs a pairwise comparison of two binary segmentations by relative overlap. Images are analysed in a pixelwise manner to calculate the total number of false positive, false negative, true positive and true negative pixels respectively.

Let $N_{P}$ be the number of false positive pixels, $N_{N}$ the number of false negative pixels, $N_{R}$ the total number of reference pixels, and $N_{S}$ the total number of subject pixels. We considered the following correspondence measure:

$$
C(S, R)=1-\frac{N_{N}+N_{P}}{N_{R}+N_{S}}, N_{S}+N_{N}=N_{R}+N_{P}
$$

This measure gives a score of 1 if subject $S$ and reference $R$ are identical and 0 if $S \cap R=\Phi$.

Figure 9 shows a representation of the correspondence measure computed for 25 images in an axial sequence. This representation highlights the high quality of the results obtained by our approach. The average value of the correspondence measure computed over the entire database is $90.11 \%$.

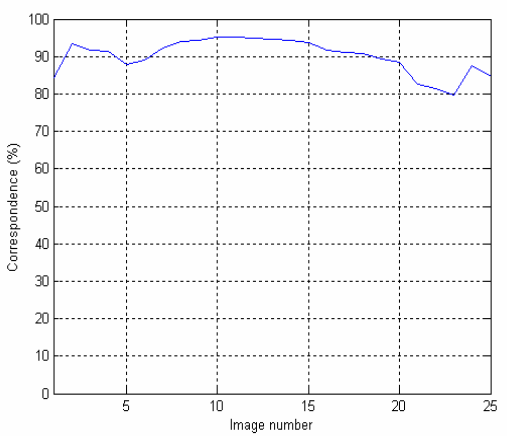

Figure 9. Correspondence measure between automatic and manual segmentations

The second evaluation procedure was performed in order to assess the quality of the 2D segmentation results for the further step in the project, namely the $3 \mathrm{D}$ reconstruction of the shoulder complex. Figure 10 shows a 3-D model of the shoulder reconstructed from an axial sequence by using the open-source software $3 d$ Slicer [13]. In order to obtain an accurate 3D model, the 2D segmentations used as input data have to be very accurate as well.

The quantitative assessment of the shoulder model was performed by computing the minimal 3D acromio-humeral distance. This computation was implemented in Polyworks, a software developed by Innovmetric Inc. and dedicated to the inspection of 3D models (see Fig. $10 \mathrm{c}$ ). The average error for the 20 models corresponding to the sequences in our database respectively was $0.56 \mathrm{~mm}$, considering as reference the average value of $5 \mathrm{~mm}$ corresponding to the normal distance in healthy patients. 


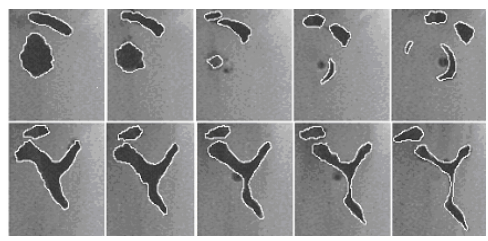

a)

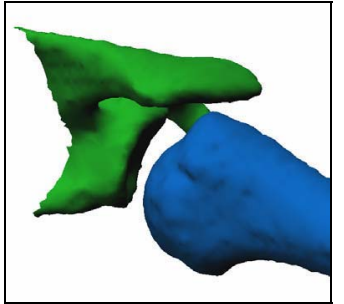

b)

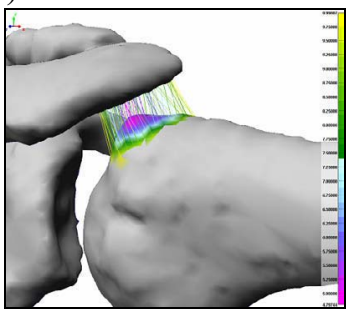

c)
Figure 10. a) sample of the 2D segmented sequence of axial images corresponding to the $3 \mathrm{D}$ model; $\mathrm{b}$ ) $3 \mathrm{D}$ reconstructed model of the shoulder complex : scapula(green) and humerus (blue) c) computation of the acromio-humeral distance in Polyworks.

\section{Conclusion and future work}

In this paper, we propose a new automatic 2D segmentation technique for the detection of the bony structures of the shoulder in functional magnetic resonance images. As shown in the previous section, our method yields excellent performances when compared to manual segmentations performed by radiologists. Moreover, the sequences of segmented images are suitable for the reconstruction of an accurate 3D shoulder model. This model will be used in the study of biomechanics and arthrocinematic of the joint shoulder complex in patients with shoulder pathologies such as instability and impingement.

Our approach is task-oriented and handles well the specific features of MR shoulder images. However, it is easily adaptable for the segmentation of other types of volumetric medical images. Indeed, the proposed technique allows for an accurate extraction of other anatomic structures with complex shapes and a reasonable degree of homogeneity.

Future work will consider texture analysis for the segmentation of soft tissues involved in the shoulder complex (muscles, tendons, ligaments, and capsule) in order to pursue the computer visionbased study of shoulder pathologies.

Acknowledgements This research was supported by a student grant from the National
Science and Engineering Research Council of Canada. Innovmetric Software Inc. has kindly provided one license for Polyworks.

\section{References}

[1] L.J. Hébert, H. Moffet, M. Dufour, and C. Moisan, Acromiohumeral distance in a seated position in persons with impingement syndrome. Journal of Magnetic Resonance Imaging, vol. 18, no.1, 2003, pp. 72-79.

[2] Z.P. Liang, P.C. Lauterbur, Principles of magnetic Resonance Imaging: A signal processing perspective,.IEEE Press Series on Biomedical Engineering, New York, 2001.

[3] H. Soltanian-Zadeh, J.P. Windham, D.J. Peck, and A.E. Yagle, A comparative analysis of several transformations for enhancement and segmentation of magnetic resonance image scene sequences. IEEE Trans. on Medical Imaging, vol. 11 , no. 3 , 1992, pp. 302-318.

[4] S. Hu, E. A. Hoffman, J. M. Reinhardt, Automatic lung segmentation for accurate quantitation of volumetric $X$-ray $C T$ images. IEEE Trans. on Medical Imaging, vol. 20, no. 6, 2001, pp. 490-498.

[5] S.D. Pathak, D.R. Haynor, and Y. Kim, Edge-guided boundary delineation in prostate ultrasound images. IEEE Trans. on Medical Imaging, 2000, vol. 19, no. 12, pp. 1211-1219.

[6] J.-P. Cocquerez and S. Philipp, Analyse d'images: filtrage et segmentation. Ed. Masson, Paris, 1995.

[7] Y. Lu, T. Jiang, and Y. Zang, Region growing method for the analysis of functional MRI data. NeuroImage, vol. 20, 003, pp. 455-465.

[8] R.C.Gonzalez and R.E Woods., Digital image processing, Prentice Hall, 2002.

[9] Y. Kang, K. Engelke, and W. A. Kalender, A new accurate and precise 3-D segmentation method for skeletal structures in volumetric CT data, IEEE Trans. on Medical Imaging, vol. 22, no. 5, 2003, pp. 586-598.

[10] R. Pohle and K.D. Toennies, A new approach for model-based adaptive region growing in medical analysis, Proc. of 9th Int. Conf. on Computer Analysis and Patterns, Warsaw, 2001, pp. 238-246.

[11] A. Branzan-Albu., D. Laurendeau, and C. Moisan, Tumour detection in MR liver images by integrating edge and region information, Proc. of Modelling \& Simulation for Computer-aided Medicine and Surgery, INRIA Rocquencourt, Nov. 12-15, 2002.

[12] G. Gerig, M. Jomier, and M. Chakos, "Valmet: A new validation tool for assessing and improving 3D object segmentation", Proc. MICCAI Conf. on Medical Image Computing and Computer-Assisted Intervention, Utrecht, 2001, pp. 516-523.

[13] D. Gering et al : An Integrated Visualization System for Surgical Planning and Guidance Using Image Fusion and an Open MR. Journal of Magnetic Resonance Imaging, Vol 13, 2001, pp. 967-975. 